\title{
ASOCIATIVIDAD, CADENA DE VALOR E IMPACTO DE AMBAS
}

\author{
ASSOCIATIVITY, VALUE AND IMPACT CHAIN OF BOTH
}

\author{
María Leonor Parrales Poveda ${ }^{1}$, Carlos Emilio Basurto Vinces ${ }^{2}$, Madelayne Ivana Cruz \\ Vidaurre ${ }^{3}$, José Alberto Ponce Prado ${ }^{4}$
}

\author{
1,2,3,4 - Universidad Estatal Del Sur De Manabí, Jipijapa, Ecuador
}

1. Email: maria.parrales@unesum.edu.ec ORCID: https://orcid.org/0000-0003-3994-3711

2. Email: basurto-carlos8565@unesum.edu.ec ORCID: https://orcid.org/0000-0001-5209-4246

3. Email: cruz-madelayne1243@unesum.edu.ec ORCID: https://orcid.org/0000-0002-9185-0419

4. Email: ponce-jose1512@unesum.edu.ec ORCID: https://orcid.org/0000-0002-2744-9778

Recibido: 11/05/2021 Aceptado: 23/07/2021

Para Citar: Parrales Poveda, M. L., Basurto Vinces, C. E., Cruz Vidaurre, M. I., \& Ponce Prado, J. A. (2021). Asociatividad, cadena de valor e impacto de ambas. Revista Publicando, 8(31), 392-413. https://doi.org/10.51528/rp.vol8.id2259

\begin{abstract}
Resumen:
El avance científico en temas como la inteligencia artificial y la biotecnología, así como la pandemia por la Covid-19 presentan desafíos a la economía mundial, especialmente a los productores y empresarios que deben con resiliencia adaptarse al momento. En Ecuador, con un sistema económico social y solidario, los emprendedores, micro, pequeños y medianos empresarios deben unir sus esfuerzos para enfrentar estos retos. Una vía es la asociatividad. Sin embargo, existe resistencia al trabajo cooperativo, impera el individualismo y la intermediación como medio para llevar la producción al consumidor. Este, es uno de los nudos críticos en su cadena de valor, que no ha permitido al productor mejorar su estatus social. Por ello, con la revisión de una considerable literatura y un trabajo de campo se explica que conformar asociaciones posibilita desarrollar proyectos más eficientes, minimiza riesgos, mejora la competitividad e incrementa la producción, consolida alianzas entre distintos agentes que interactúan en el mercado, promoviendo oportunidades de crecimiento individual y colectivo. La asociatividad articulada a la cadena de valor permite desarrollar ventajas competitivas y crear valor a su marca.
\end{abstract}

Palabras clave: asociatividad, cadena productiva, capital social, trabajo en equipo, ventaja competitiva.

\begin{abstract}
:
Scientific advances in areas such as artificial intelligence and biotechnology, as well as the Covid-19 pandemic, present challenges to the world economy, especially to producers and entrepreneurs who must resiliently adapt to the moment. In Ecuador, with a social and solidarity-based economic system, entrepreneurs, micro, small and medium-sized businesses must unite their efforts to face these challenges. One way is through associativity. However, there is resistance to cooperative work, individualism and intermediation as a means to bring production to the consumer prevails. This is one of the critical knots in the value chain, which has not allowed producers to improve their social status. Therefore, with the review of considerable literature and field work, it is explained that forming associations makes it possible to develop more efficient projects, minimizes risks, improves competitiveness and increases production, consolidates alliances between different agents that interact in the market, promoting opportunities for individual and collective growth. The associativity articulated to the value chain makes it possible to develop competitive advantages and create value to its brand.
\end{abstract}

Keywords: associativity, productive chain, social capital, teamwork, competitive advantage. 


\section{INTRODUCCIÓN}

$\mathrm{U}$ no de los puntos clave dentro del desarrollo de la competitividad a nivel mundial, se basa en lograr la ansiada economía de escala y obtener poder de negociación frente a los clientes. Uno de los problemas cruciales para llegar a ello lo representa el limitado recurso con el que cuentan los pequeños productores, los cuales de manera individual ven disminuidas sus posibilidades $\mathrm{y}$ tienden a ser desplazados al último lugar de protagonismo en la cadena productiva o de valor, lo que en respuesta promovió la asociatividad, establecida a nivel organizacional con el fin de formar un eslabón más fuerte que les permita a las micro, pequeñas y medianas empresas (Mipymes) poder negociar en bloque, tanto con proveedores como con los clientes, logrando así, mejorar la calidad y el precio de sus productos.

En ese sentido una asociación, responde a un grupo de personas que con un objetivo en común deciden unirse para enfrentar la producción, transformación, comercialización y postventa de un producto. Según la teoría de Katzenbach y Smith (1993) definen el trabajo en equipo como un grupo pequeño de gente con habilidades complementarias quienes están comprometidos a un propósito común, metas de rendimiento y enfoque por lo cual ellos son mutuamente responsables de rendir cuentas. Esta simple definición trae consigo modelos a seguir tales como Belbin y Margerison-McCann (habilidades complementarias), gestión de proyectos con P3 (propósito común y metas de rendimiento) y modelos de liderazgo tales como Hersey y Blanchard y McGregor (mutualmente responsables por cuentas).
Entre las muchas observaciones sobre equipos, Katzenbach y Smith (1993) identificaron cinco niveles de trabajo de equipo como grupo de trabajo (se comparte información sin metas claras); falso equipo (no actúan como unidad por miedo al riesgo); equipo potencial (meta en común con responsabilidad contable mutua); equipo real (propósito común y enfoque. Comparten responsabilidad en cuentas por los resultados) y equipo de alto rendimiento (miembros están comprometidos los unos con los otros acerca de su crecimiento personal y su desarrollo). En asociatividad es necesario integrarse con responsabilidad y metas claras que beneficien a todos los miembros.

No obstante, la idea de la asociatividad es muchas veces subestimada por muchos empresarios quienes por falta de conocimiento no logran ver la gran oportunidad que les puede ofrecer el trabajo mediante este sistema, el cual, siendo bien aplicado y en las condiciones adecuadas, puede ser altamente provechoso en diversos aspectos inclusive en el gran reto de lograr la auto sostenibilidad e independencia empresarial, que en un futuro les permita articularse comercialmente y en forma directa con el mercado internacional.

Destáquese a través de esto que, las relaciones asociativas pueden tener su origen en una amplia gama de objetivos que van desde simples uniones para operaciones concretas, hasta uniones perdurables en el tiempo para operaciones recurrentes; estas relaciones en la práctica pueden ir desde la informalidad hasta la constitución de figuras jurídicas específicas. De allí, que, en los 
últimos años, el movimiento asociativo se ha venido desarrollando, despertando el interés de diferentes actores, generando una amplia gama de relaciones inter empresariales, entre las que se pueden señalar relaciones de empresas con proveedores; con clientes; Mipymes, Organizaciones de Economía Popular y Solidarias (OEPS); Mipymes con instituciones intermedias; Mipymes y el Estado, entre otras.

En la mayoría de los países de América Latina, el concepto de asociatividad se adopta según los criterios que la Ley establezca, no obstante, aspectos como el volumen de ventas y el número de trabajadores son los más comunes a la hora de establecer tal clasificación. Por su parte de acuerdo con lo que afirma Otoya (2005), la pequeña empresa es:

"La unidad económica constituida por una persona natural o jurídica, bajo cualquier forma de organización o gestión empresarial contemplada en la legislación vigente y que tenga por objeto producción, comercialización de bienes o prestación de servicios" (p.32).

A tal efecto, Rosales (2016) define a la asociatividad como:

"Un mecanismo de cooperación entre empresas pequeñas y medianas, en donde cada conjunto participante, manteniendo su independencia jurídica y autonomía gerencial, decide voluntariamente participar en un esfuerzo conjunto con los otros participantes para la búsqueda de un objetivo común" (p. 24).

Lo señalado, implica que cuando dos o más personas o empresas se asocian lo que buscan es unir esfuerzos para alcanzar objetivos que son comunes y que por sí solos no es posible conseguir. Por su parte, la asociatividad empresarial representa un mecanismo mediante el cual las organizaciones unen voluntades, iniciativas y recursos, alrededor de objetivos comunes, con el propósito de ser más competitivas en el mercado global.

Es así como, la asociatividad es vista como:

"Serie de coaliciones formales entre dos o más organizaciones, que permiten llevar a cabo empresas en el corto plazo, originadas en relaciones oportunistas o permanentes que se desarrollan como una forma de sociedad entre los participantes" (Rosales, 2016, p.44).

De ahí, que la asociatividad es por tanto una herramienta que busca contrarrestar consecuencias negativas en el momento de aliarse con otras empresas para conseguir metas similares y objetivos comunes.

En otras palabras, la asociatividad representa un mecanismo de cooperación entre Mipymes y/o OEPS, en donde cada conjunto participante, manteniendo su independencia jurídica $y$ autonomía en la toma de decisiones, decide voluntariamente participar en un esfuerzo conjunto con otros participantes en la búsqueda de una meta en común, lo que implica la interacción de las personas o bien de empresas en una red de intercambios, este intercambio difiere dependiendo del objeto que persigan los involucrados en dicha interacción, con el propósito de unir esfuerzos para alcanzar proyectos similares que individualmente no se conseguirían.

Por ello, es de notar que el impacto generado por la sociabilidad espontánea sobre la vida económica es realmente muy significativo, considerándose que este proceso de asociatividad 
se basa en el resultado natural de la consolidación del capital social al interior de los grupos y se manifiesta cuando dos o más personas se interrelacionan para lograr propósitos comunes. Es así, como la asociatividad puede definirse como la tendencia de los seres humanos a agruparse para convivir, resolver problemas y defenderse frente a amenazas reales, siendo una de sus fortalezas su estrecha relación con el concepto de capital social y el grado de desarrollo del mismo (Swans, 2015).

En términos generales, la asociatividad, representa un tipo de organización social, conformada por sujetos que, fundados en diferentes tipos de acuerdos, deciden unirse de manera permanente y voluntaria para compartir sus esfuerzos y conocimientos en la consecución de un objetivo común. Estos acuerdos son el resultado de la interacción social que los sujetos ponen en práctica en el día a día, estando mediados por una diversidad de códigos compartidos o estatutos asociativos, los cuales en consecuencia generan y establecen la cadena de valor, lográndose con ello un impacto gerencial de gran valor para la economía de estas Mipymes.

\section{Modelo de Asociatividad: ¿Para qué asociarse?}

\section{Figura 1}

Modelo de Asociatividad para las Mipymes.

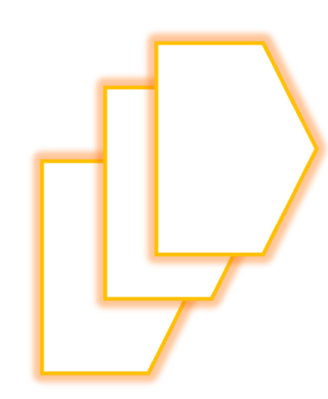

Cadenas de valor de los proveedores

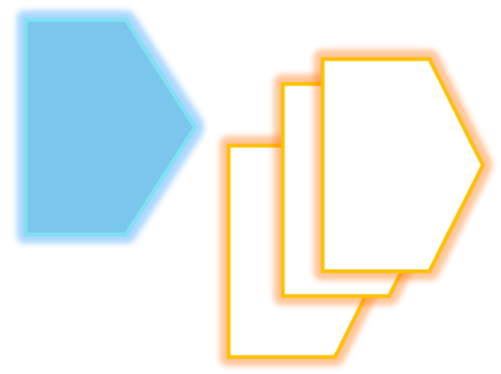

Cadenas de valor de la empresa
Cadenas de valor del canal

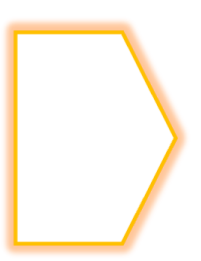

Cadenas de valor de los compradores

Fuente: Michael Porter. Revista: La Ventaja Competitiva de las Naciones (1995).

El sistema de valor referido identifica la importancia de cada uno de los actores que participan en los procesos de asociatividad, a fin de que los productos y servicios lleguen al consumidor final. A través de él, se puede ver claramente el rol de cado uno de ellos y se puede tomar decisiones más acertadas en relación con los mismos, asumiendo que en la cadena de valor, las empresas pueden alcanzar liderazgo en costos y diferenciación en la medida que optimicen los recursos para cada una de las actividades dentro del proceso, considerándose que, entre más conectada se encuentre la cadena con todos los procesos, mayor valor alcanzará y lo que finalmente mostrará tal valor, es lo que el consumidor final esté dispuesto a pagar por ello. 
Es conocido, que las Mipymes juegan un papel determinante en la economía de un país; éstas constituyen un factor importante para el crecimiento socioeconómico de cada nación pues la conforman emprendedores, microempresarios y comerciantes que invierten con un capital limitado. En la economía ecuatoriana, según Ron Amores y Sacoto Castillo (2017) las pequeñas y medianas empresas, aportan con el 90\% al sector empresarial, el 50\% al Producto Interno Bruto y el $60 \%$ en la generación de empleo, por lo que, dinamizan todo el aparato productivo, generando riqueza, crecimiento económico y bienestar social.

Por otro lado, un sector fundamental para el desarrollo ecuatoriano es el de las OEPS, que con base en el Artículo 283 de la Constitución establece que el sistema económico responde a una economía social y solidaria y está integrado por las formas de organización pública, privada, mixta y de economía popular y solidaria.

Desde el año 2011, se reconoce, a través de la Ley Orgánica de Economía Popular y Solidaria (LOEPS), a las OEPS como una forma de organización económica en la que sus integrantes, ya sea individual y colectivamente, organizan y desarrollan procesos de producción, intercambio, comercialización, financiamiento y consumo de bienes y servicios mediante relaciones basadas en la solidaridad, cooperación y reciprocidad, situando al ser humano como sujeto y fin de su actividad, orientada al buen vivir, en armonía con la naturaleza, por sobre la apropiación, el lucro y la acumulación de capital (Art. 1).

De acuerdo con el catastro de la Superintendencia de Economía Popular y Solidaria (SEPS), al 15 de agosto de 2020 existen 16.811 OEPS:

Tabla 1. Distribución de OEPS por sector

\begin{tabular}{lc}
\hline SECTOR & $\begin{array}{c}\text { NÚMERO DE } \\
\text { ORGANIZACIONES }\end{array}$ \\
\hline Asociativo & 13616 \\
Cooperativo & 3035 \\
Comunitario & 62 \\
Federaciones & 5 \\
Redes & 41 \\
Uniones & 52 \\
TOTAL & 16811 \\
\hline
\end{tabular}

Fuente: Base de datos de la SEPS (15 de agosto de 2020) Elaboración: Dirección de Fortalecimiento de Actores (DFA)-IEPS

El asociacionismo es la particularidad de las OEPS, entendida como un nivel secundario de solidaridad que excede al del grupo doméstico o unidad doméstica que es la célula de la economía popular. La mayoría representada por el sector asociativo. En lo que respecta a las cooperativas la mayor concentración es para el servicio de transporte. 
Figura 2

Distribución de OEPS por sector.

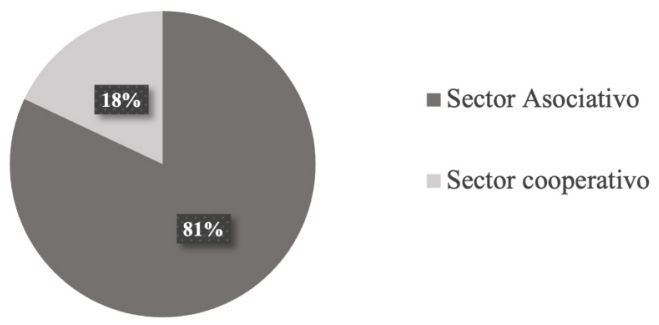

Fuente: Base de datos de la SEPS (15 de agosto de 2020) Elaboración: Dirección de Fortalecimiento de Actores (DFA)-IEPS.

En Ecuador, las OEPS se encuentran insertas en una infinidad de actividades económicas, tales como, agricultura, ganadería, caza y silvicultura, construcción, enseñanza, explotación de minas y canteras, manufactura, pesca, reciclaje, servicios, transporte, almacenamiento y comunicación, turismo y otras. Pero a diferencia de las empresas de capital, intentan satisfacer necesidades con base en prácticas de solidaridad, equidad, autogestión, cooperación, etc.

En el sector agrícola, ganadero y pesquero de la Economía Popular y Solidaria (EPS) en el Ecuador según la Encuesta Nacional Empleo, Desempleo y Subempleo (ENEMDU) a diciembre de 2019 abarca a 2'290.304 personas. Con respecto a su facturación declarada de 9,5 millones de USD en el año 2010 pasó a 237,9 millones de USD en el año 2018 que corresponden al sector de la EPS. Las organizaciones de la EPS que se dedican a estas actividades económicas están presentes en las provincias de Los Ríos, Manabí, Guayas, El Oro, Chimborazo, Tungurahua y Loja (IEPS, 2020).

Desde esta perspectiva, se destaca que la asociación entre productores tiene carácter comercial o industrial, se crean con miras a defender intereses del mercado interno, que han derivado hacia el mercado externo mediante el establecimiento de un servicio de exportación, al cual se confían las actividades de comercialización externa. Si bien existen como un departamento de la asociación patrocinadora, suele tener una atracción independiente formada en muchos casos por un consejo de administración y un gerente, con personal operativo cedido en la mayoría de los casos por la asociación. Entre las etapas del proceso asociativo se encuentran algunas fases a criterios de Liendo y Martínez (2001):

1. Etapa de gestación: Durante este período se despierta el interés de los participantes al iniciarse un proceso de acercamiento. Se analizan fortalezas de las unidades productivas y las ventajas a lograr si se concreta el proyecto.

2. Etapa de estructuración: En esta etapa, los empresarios ya han definido su rol dentro del grupo y han aceptado al mismo como herramienta para alcanzar los objetivos planteados. En este momento se definen las estrategias a seguir para el logro de los objetivos comunes.

3. Etapa de madurez: A esta altura del proceso de desarrollo, los empresarios ya han definido pautas de organización del grupo, el mismo ha adquirido identidad como tal y existe claridad sobre las acciones a seguir. En esta etapa se define la forma jurídica, basándose en las necesidades planteadas por la actividad a desarrollar. Así se crean las bases para la auto sostenibilidad del grupo.

4. Etapa productiva o de gestión: Este es el período donde se llevan a cabo las acciones para la obtención de los resultados esperados. Los 
procesos operativos internos se agilizan para llevar adelante la gestión empresarial.

5. Etapa de declinación: Esta etapa comienza cuando los rendimientos de la gestión disminuyen y la relación costo-beneficio del accionar del grupo es negativa. Es aquí donde deberá tomarse la decisión de desintegrar el grupo o iniciar nuevos proyectos.

En este sentido, la asociatividad se constituye como un mecanismo de solidaridad que resulta ser una alternativa para mejorar la calidad de vida de las personas. Estas presentan varias ventajas: Principalmente se caracterizan por la conformación de grupos asociativos que permiten resolver problemas conjuntos manteniendo la autonomía gerencial de las empresas participantes, este convenio organizacional puede adoptar diversas modalidades jurídicas y organizacionales, no excluye a ninguna empresa por el tipo de mercado en el cual opera, es exclusiva para Mipymes.

Mediante la asociatividad se pueden generar alianzas estratégicas, franquicias, subcontrataciones con grandes empresas donde cada una tenga un papel importante y puedan lograr un gana-gana sin que ninguno salga afectado; también, puede convertirse en una de las herramientas utilizadas por las Mipymes para afrontar la apertura de nuevos mercados y entender mejor la globalización y tendencias que dado el momento vive el mundo. Uno de ellos es resistir la pandemia que por coronavirus enfrentan las naciones, en donde la mentalidad abierta, capacidad de resiliencia, manejo de internet $y$ redes sociales han permitido un intercambio de experiencias que han dado sostenibilidad a muchos pequeños negocios en diferentes esferas. Pero hay un problema, la resistencia a trabajar en grupos cooperativos.

Según Hurtado (2007) en Ecuador, existe una cultura anti-asociativa, lo que conlleva al fracaso de muchas pequeñas y medianas empresas, comercios y emprendimientos, fracaso que va ligado a su tamaño y dificultades operativas.

De ahí que, la crisis por la emergencia sanitaria puso en riesgo a los pequeños productores de las zonas urbanas y rurales que son uno de los sectores generadores de empleo y fuentes de ingresos de las familias. Es bien sabido que las Mipymes y las OEPS se han visto afectadas severamente por la Covid-19, especialmente porque tienen menos activos, son particularmente dependientes de sus transacciones diarias y de reservas de efectivo más condicionadas que las grandes empresas. A raíz de la pandemia muchas paralizaron sus actividades económicas, cerraron sus actividades o redujeron significativamente su producción. La falta de liquidez y financiamiento fue un factor clave que ocasionó que muchas no se mantengan en pie, las que lo han hecho diversificaron su producción, exploraron la actividad textil, donde la fabricación de mascarillas, trajes de bioseguridad y otros implementos ha representado una nueva fuente de ingresos que contribuye a la sostenibilidad de las OEPS.

La asociatividad agrupa las formas más sofisticadas y avanzadas del llamado proceso de integración empresarial, su puesta en marcha fomenta el desarrollo de las empresas y sectores empresariales que las integran impulsando el crecimiento económico y la capacidad competitiva 
de las localidades, la incorporación de tecnología, mejor posición para negociar con clientes y proveedores, reducir costos, mejora en el posicionamiento de marca y manejo de economía circular, entre otras.

En resumen, mediante la asociatividad se producen movimientos que aceleran los procesos que incentiva a las empresas participantes a aportar sus conocimientos, habilidades y métodos de producción y en esta dinámica se fortalecen y generan crecimiento y estar mejor preparados para la batalla de la competitividad.

Las autoras Parrales y Rodríguez (2019) manifiestan que la asociatividad y la cadena de valor son factores importantes para hacer frente al desarrollo económico. Un caso particular de asociatividad es de la Asociación de Productores Orgánicos de Vinces (APOVINCES), que opera desde el año 2006. Está conformada por campesinos dueños de terrenos de máximo ocho ha y que aglutina a casi 350 socios legalizados de los cantones Baba, Palenque, Pueblo viejo y el mismo Vinces, además de 300 a 400 productores en tránsito. Su finalidad es la "Autogestión y fortalecimiento asociativo de base familiar campesina en la producción de cacao", compiten con trasnacionales de producción confitera $y$ comercializadoras privadas de cacao, la organización campesina impone el precio tope del quintal de cacao en la zona, ha logrado negociar y vender directamente con empresas suizas, empresas que se ven atraídas por las certificaciones que ostentan (Clarket al., 2017).

\section{Cadena de valor $y$ asociatividad}

Una de las grandes ventajas que se obtiene con la asociatividad es el mayor control en la cadena de valor (Viloria, 2013).

El concepto de las cadenas de valor se remonta a los años 60 y 70 cuando Raphael Kaplisky utiliza este concepto como herramienta para indagar sobre los cambios que se generan en la distribución de las ganancias por el proceso de globalización. Según Kaplisky (citado por Alvarado, 2016) las cadenas de valor describen el rango completo de las necesidades que son necesarias para llevar el producto o servicio por todas las etapas respectivas hasta llegar al consumidor final.

Lo anterior implica que las etapas necesarias para producir un servicio constituyen los eslabones en una cadena de servicios. La cadena de valor, además, es un tema que está muy asociado al marketing de servicios, dado que todo el proceso que se realiza para llevar los productos y servicios hasta el cliente final constituyen una serie de actividades en cadena que deben generar beneficio especialmente para el cliente y posicionamiento de marca.

Según Benítez, las cadenas productivas son "un conjunto de agentes económicos que participan directamente en la producción, transformación, comercialización y en el traslado hacia el mercado del mismo producto" (2015, p.32).

La cadena de valor, permite localizar a las empresas, las instituciones, las operaciones, las dimensiones y la capacidad de negociación, las tecnologías, las relaciones de producción y relaciones de poder en la determinación del precio. Estas cadenas a su vez responden a ciertas tipologías de acuerdo con su enfoque. 


\section{Figura 3}

Actividades Primarias de la Cadena de Valor.

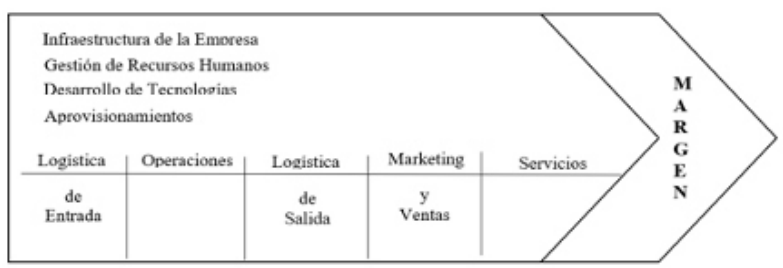

Fuente: Michael Porter. Revista: La Ventaja Competitiva de las Naciones, 1995.

En el desarrollo de estas actividades, se generan los sistemas de valor que son la suma de todas ellas, este sistema de valor está representado en un conjunto de diferentes tareas donde participan varios actores con roles bien definidos. Entre más valor cree una empresa en sus actividades, mayor valor se generará en los sistemas teniendo en cuenta el manejo óptimo de los enlaces en cada sistema. La ventaja competitiva, va de la mano de la cadena de valor, dado que para que se produzca es necesario que las actividades se gestionen como un sistema y no como partes independientes. El todo coherente está implícito en toda la cadena productiva.

Para Padilla, los eslabones que conforman la cadena de valor como "eslabones verticales", son lo que pueden proporcionar oportunidades para que la empresa aumente su ventaja competitiva" (Padilla, 2014, p.81). Asimismo, señala que es con frecuencia posible beneficiar tanto a los otros actores de la cadena como a la empresa involucrada al influir la configuración de las cadenas de valor de los demás participantes para que juntos optimicen el desempeño de las actividades o mejorando la coordinación entre las cadenas de la empresa y de los compradores.
El valor según Porter (1995) es creado cuando una empresa crea una ventaja competitiva para su comprador, disminuyéndole el costo, aumentando su nivel de satisfacción o cumpliendo sus necesidades" (p.11). De allí, que el valor creado para el comprador debe ser percibido por este si se quiere recompensar con un precio mínimo, lo que significa que las empresas deben de comunicar sus valores a los compradores a través de medios de publicidad y fuerza de ventas. La satisfacción de necesidades del comprador implica con frecuencia el coordinar actividades en conjunto, con lo cual se logra mayor eficiencia entre ambas partes.

Desde la teoría general se ha destacado la importancia de la cadena de valor en la producción. Esta es una herramienta de análisis que sirve para planificar y se divide en actividades primarias y secundarias. Las primarias contienen cinco subactividades que se enfocan en la creación del producto, su venta y postventa. Las actividades secundarias se subdividen en infraestructura, gestión del talento humano, desarrollo de tecnología y compras, todas sirven de apoyo a las actividades primarias. Su conocimiento, permite a la empresa crear valor y con ello destacar su ventaja competitiva que lleva a los involucrados a posicionarse mejor en el mercado y sobre todo en la mente del consumidor final.

\section{MATERIALES Y MÉTODOS}

La presente investigación reúne las condiciones metodológicas de una investigación documental y de campo, puesto que se han utilizado conocimientos de ideas previas dirigidas a analizar la "Asociatividad, Cadena de Valor y el impacto de ambas", para así, finalmente elaborar un cuerpo de consideraciones generales que ayuden a ampliar el 
interés propuesto y dar continuidad a una nueva investigación, dados los resultados obtenidos.

El alcance de la investigación es correlacional porque investiga y mide la relación entre la asociatividad como modelo de gestión empresarial y la cadena de valor de los habitantes dedicados a la actividad agrícola, la cual es sustentada y/o comprobada a través del coeficiente de correlación Chi-cuadrado de Pearson (relación estadística). Se usó el método inductivo, para responder con eficiencia a las necesidades investigativas y llevar a cabo una valoración de los hechos de forma particular para llegar la explicación desde una visión general.

Por otro lado, el enfoque de la investigación es cuantitativo. Es importante mencionar que, el enfoque cuantitativo surgió a partir del análisis estadístico que se realizó tras la aplicación de encuestas a una muestra intencional de 193 productores agrícolas de la parroquia El Anegado. El cuestionario se estructuró con diecinueve preguntas dicotómicas y sus resultados arrojaron insumos para identificar la disposición de los productores agrícolas para emprender una estrategia de asociatividad empresarial, también reveló elementos necesarios para el diseño del modelo asociativo, que permitió probar la hipótesis planteada, la relación entre las variables y posteriormente, formular las respectivas conclusiones.

Se utilizó la técnica del subrayado y el fichaje, en la revisión de documentos se encontraron ideas explícitas relacionadas con los tópicos encargados de identificar a cada característica insertada en el estudio. Por lo tanto, se realizaron continuas interpretaciones con el claro propósito de revisar aquellas apreciaciones o investigaciones propuestas por diferentes investigadores relacionadas con el tema de interés, para luego dar la respectiva respuesta a los planteamientos, en función de las necesidades encontradas en la indagación y al lugar objeto de estudio. Para Arias (2010), las fuentes documentales incorporadas en la investigación documental o bibliográfica, "representa la suma de materiales sistemáticos que son revisados en forma rigurosa y profunda para llegar a un análisis del fenómeno" (p.41). Por lo tanto, se procedió a cumplir con la realización de una lectura previa determinada por encontrar aquellos aspectos estrechamente vinculados con el tema, para luego explicar mediante un desarrollo las respectivas apreciaciones generales de importancia.

Para orientar el proceso de investigación y como parte básica de selección de documentos que se presentan en el contenido teórico se analizaron los siguientes documentos detallados a continuación (Ver la próxima página). 
Tabla 2: Literatura analizada

\begin{tabular}{lll}
\hline Autor/ año & Título & Publicación \\
\hline Alvarado, Y. (2016) & Cadena de valor Empresarial. & $\begin{array}{l}\text { Foro Regional sobre Política } \\
\text { Industrial-Santa Cruz de la } \\
\text { Sierra, 23. }\end{array}$ \\
\hline Benitez, C. (2015) & $\begin{array}{l}\text { Cadena de Valor y su } \\
\text { impacto en la asociatividad. }\end{array}$ & $\begin{array}{l}\text { Cuaderno de desarrollo } \\
\text { económico No 3 Universidad de } \\
\text { La Plata, 32. }\end{array}$ \\
\hline Iguera, R. (2012) & Asociatividad entre PYMES. & $\begin{array}{l}\text { Revista de Economía de la } \\
\text { Universidad de Zulia en } \\
\text { Maracaibo, Caracas, 14. }\end{array}$ \\
\hline $\begin{array}{l}\text { Katzenbach, J. R. y } \\
\text { Smith, D.K. (1993) }\end{array}$ & $\begin{array}{l}\text { The Wisdom of Teams: } \\
\text { Creating the High- }\end{array}$ & $\begin{array}{l}\text { Harvard Business School, } \\
\text { performance Organization. }\end{array}$ \\
\hline & & $\begin{array}{l}\text { Sextas Jornadas "Investigaciones } \\
\text { en la Facultad" de Ciencias } \\
\text { Económicas y Estadística. } \\
\text { Instituto de Investigaciones } \\
\text { Económicas, Escuela de }\end{array}$ \\
$\begin{array}{l}\text { Liendo, M. y Martinez, } \\
\text { A. (2001) }\end{array}$ & $\begin{array}{l}\text { Asociatividad una alternativa } \\
\text { para el desarrollo y } \\
\text { crecimiento de las Pymes. }\end{array}$ & $\begin{array}{l}\text { Economía. Universidad Nacional } \\
\text { de Rosario. }\end{array}$ \\
& &
\end{tabular}

Fortalecimiento de las cadenas de valor como

Padilla, R. (2014) instrumento de la política industrial: Metodología y CEPAL experiencia de la CEPAL en

Centroamérica.

\begin{tabular}{lll}
\hline $\begin{array}{l}\text { Parrales, M. y } \\
\text { Rodríguez, K. (2019) }\end{array}$ & $\begin{array}{l}\text { Asociatividad y cadena de } \\
\text { valor frente al crecimiento } \\
\text { económico. }\end{array}$ & $\begin{array}{l}\text { II Congreso Internacional de } \\
\text { Gerencia y Gestión Productiva } \\
\text { \#CI2GP 2019. Pontificia } \\
\text { Universidad Católica de } \\
\text { Ecuador, sede Manabí. }\end{array}$ \\
\hline Porter, M. (1995) & $\begin{array}{l}\text { Las PYMES. Asociatividad y } \\
\text { su impacto en el mundo } \\
\text { competitivo de las } \\
\text { organizaciones. }\end{array}$ & $\begin{array}{l}\text { Cuaderno de desarrollo } \\
\text { económico No 3 Universidad de } \\
\text { La Plata, 11. }\end{array}$ \\
\hline Rosales, D. (2016) & $\begin{array}{l}\text { La competitividad de las } \\
\text { PYMES. }\end{array}$ & $\begin{array}{l}\text { Revista de Economía de Perú. } \\
\text { Seminario Internacional } \\
\text { Desafios, 24. }\end{array}$ \\
\hline Swans, G. (2015) & $\begin{array}{l}\text { Asociatividad y } \\
\text { Productividad un eje para el } \\
\text { desarrollo de la } \\
\text { competitividad. }\end{array}$ & $\begin{array}{l}\text { Artículo empresarial del Boletín } \\
\text { documentación de - El Salvador, } \\
\text { 32. }\end{array}$ \\
\hline Viloria, M. (2013) & Asociatividad Empresarial. & $\begin{array}{l}\text { PRODES Programa de la } \\
\text { Universidad de España, 18. }\end{array}$ \\
\hline
\end{tabular}




\section{RESULTADOS}

La encuesta aplicada en el contexto de los productores agrícolas de la parroquia El Anegado donde se cultiva principalmente el maíz, fréjol, café, plátano, permite extraer los siguientes resultados sobre la asociatividad y la cadena de valor.

Se han identificado organizaciones, asociaciones y comités dedicados a actividades sociales, económicas, productivas, de las cuales muchas de ellas se encuentran inactivas. Por ello inicialmente se pretendió conocer si la muestra intencionada de productores (193) forma parte de un grupo asociativo para lo cual se obtuvo que el 93\% de los productores agrícolas no forma parte de ningún grupo asociativo, solo el $7 \%$ pertenece a una forma de asociación, entre ellas gremio, comité y cooperativas (véase figura 4).

\section{Figura 4}

Actualmente forma parte de una asociación.

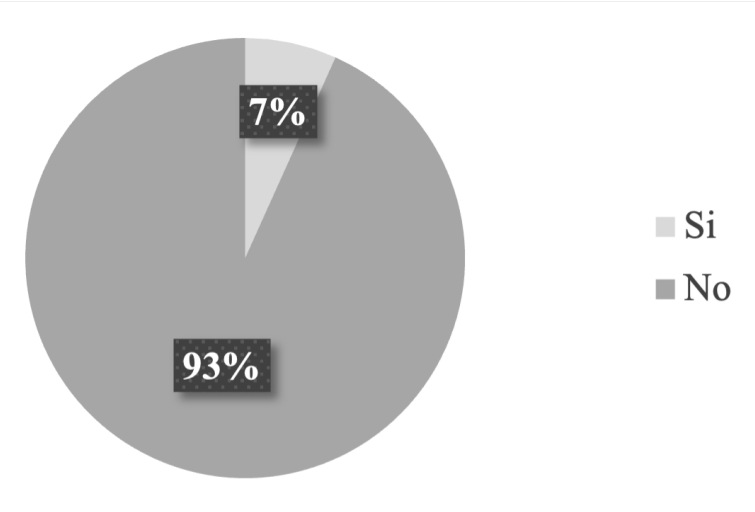

Nota: Elaborado por los Autores.

Este resultado invita a la reflexión de ¿Por qué a pesar de que la asociatividad es un mecanismo que permite a las Mipymes y OEPS tener beneficios, los productores agrícolas de la parroquia El Anegado no se han asociado? En ese sentido, la localidad objeto de análisis presenta algunos grupos asociativos como la Organización AAPAGRIM, legalmente constituida y dedicada al cultivo y producción del café tostado y molido, miel de abeja y balanceados. Cuenta con 135 socios; por otro lado, está la asociación de Emprendedores Productores El Anegado, dedicada a la producción y cultivo varios, cuenta con 90 socios. También existe la asociación Agropecuaria Flor del Salto, posee vida jurídica, cuenta con 21 socios y se dedica a actividades productivas.

La respuesta ante esta interrogante está enmarcada en varias razones, el 53\% de los productores trabajan individualmente, el 13\% desconoce los beneficios que se podrían obtener asociándose, un $10 \%$ tiene miedo a que una vez asociado los ingresos obtenidos no se distribuyan equitativamente, otro $10 \%$ mencionó que a raíz del individualismo que existe, no hay cooperación y otro porcentaje de productores no se asocian por razones como: miedo a no perseguir los mismos objetivos, miedo a la competencia, falta de recursos y conformismo (véase figura 5). Se puede llegar a deducir por medio de los resultados obtenidos que la razón principal por la cual los productores no han formado parte de una asociación es por la sencilla razón de que prefieren trabajar solos. Se necesita que los productores sean capacitados en este aspecto. La asociatividad es una de las transformaciones requeridas por las nuevas condiciones de la economía ante la crisis sanitaria por la Covid-19, con el fin de superar la insuficiencia de escalas individuales. 
Figura 5

Razones por las que nunca ha formado parte de una asociación de productores

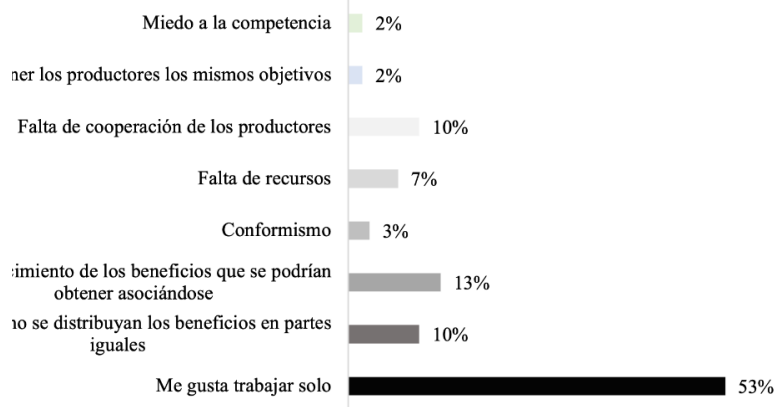

Nota: Elaborado por los Autores.

Hoy en día en un mundo cada vez más globalizado se busca un mayor nivel de competitividad por parte de los países y las diferentes organizaciones que los componen. De ahí que, en el ámbito empresarial, la supervivencia y desarrollo de cada organización va a depender de su capacidad de resiliencia y de la adaptación y respuesta que se tengan a los nuevos requerimientos de la sociedad, tales como identidad de marca, solidez, eficiencia, eficacia, responsabilidad social corporativa y procesos "amigables" con el medio ambiente, entre otros.

Los productores agrícolas de la parroquia El Anegado del cantón Jipijapa según los resultados de la encuesta tienen un gran amor a trabajar solos, están alineados al individualismo, el deseo de cooperación es casi nulo, en parte porque desconocen cuáles son los beneficios que podrían obtener al asociarse. De ahí es, que para conocer si la actitud de no asociarse depende del conocimiento de las bondades que ofrece la asociatividad se estable una relación Chi-cuadrado de Pearson.

Tabla 3. Prueba de chi-cuadrado ¿Actualmente forma parte de una asociación? *Al conocer beneficios que se podrian obtener asociandose, ¿se asociaria?

\begin{tabular}{|c|c|c|c|}
\hline & Valor & df & $\begin{array}{l}\text { Significación } \\
\text { asintótica (bilateral) }\end{array}$ \\
\hline Chi-cuadrado de Pearson & $44,868^{\mathrm{a}}$ & 1 & 0,000 \\
\hline Corrección de continuidad ${ }^{\mathrm{b}}$ & 41,835 & 1 & 0,000 \\
\hline Razón de verosimilitud & 51,562 & 1 & 0,000 \\
\hline Asociación lineal por lineal & 44,636 & 1 & 0,000 \\
\hline $\mathrm{N}$ de casos válidos & 193 & & \\
\hline
\end{tabular}

a. 0 casillas $(0,0 \%)$ han esperado un recuento menor que 5 . El recuento mínimo esperado es 8,46 . 
Con un nivel de significancia de 0,000 <0,05\% se estable una dependencia entre las variables. El hecho de que el 93\% de la muestra intencionada de productores no esté asociado es debido al escaso nivel de conocimiento que tiene sobre la asociatividad. Capacitarlos en el tema, generar mayor confianza, una visión unificada a más largo plazo, mayor integración, positivismo $y$ dinamismo en el trabajo de alto rendimiento es de vital importancia.

En ese mismo contexto, cada vez es más difícil para las Mipymes actuar de manera individual, dado su insuficiente tamaño y sus dificultades operativas, poder alcanzar resultados exitosos. Bajo esta perspectiva, las Mipymes para adecuarse a la realidad imperante se ven obligadas, en muchos casos, no sólo a encarar un proceso de introspección sino además a interactuar con el medio a través de la conformación de redes o asociaciones que los ubique en posiciones más sólidas para afrontar las amenazas y aprovechar las oportunidades. El modelo asociativo surge como uno de los mecanismos de cooperación que persigue la creación de valor a través de la solución de problemas comunes originados fundamentalmente, por falta de escala (Liendo y Martínez, 2001). Es decir, la insuficiencia del tamaño de las empresas hace muy difícil la incorporación de tecnologías, la penetración a nuevos mercados y el acceso a la información, entre otros.

Estos problemas se evidencian en el trabajo individual que realizan los productores de la parroquia El anegado. El hecho de no asociarse les ha limitado el tener acceso a capacitaciones, financiamiento, tecnologías, acceso a tierras fértiles adecuadas para la producción (véase figura 6).

\section{Figura 6}

Problemas a los que se enfrentan los productores agrícolas al trabajar individualmente.

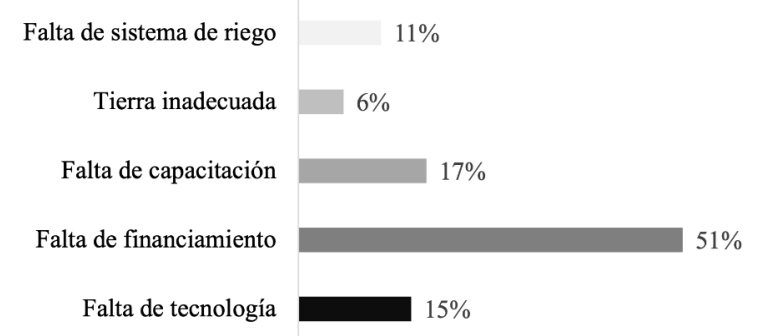

Nota: Elaborado por los Autores.

El $51 \%$ de los productores agrícolas se enfrentan a un grave problema que no les permite adquirir los insumos necesarios para mejorar y ampliar su producción. El hecho de trabajar solos les limita la posibilidad de acceder a créditos asociativos. El $17 \%$ no puede beneficiarse de capacitaciones ofertadas por entes gubernamentales. El 15\% no puede adquirir tecnologías y modernizarse. El $11 \%$ por falta de recursos económicos no tiene sistema de riego tecnificado, ocasionando que el agricultor solo realice sus actividades agrícolas por temporadas (época invernal). Un 6\% de productores no ha podido acceder a tierras fértiles para obtener una buena producción, esto hace que muchos sólo recuperen su inversión. Son muy pocos los que obtienen rentabilidad en la inversión y con ello satisfacen sus necesidades básicas. Otros ni siquiera recuperan su inversión.

Asimismo, en una economía mundial cambiante, en medio de crisis financieras, precios volátiles de los productos agrícolas y los alimentos básicos, así como los peligros del cambio climático, la financiación rural inclusiva sigue 
siendo un elemento fundamental en la transformación rural. Las instituciones financieras suelen considerar que la agricultura en pequeña escala es demasiado arriesgada y se resisten a prestar dinero a los agricultores. Otro de los desafíos es que los agricultores se resisten a solicitar préstamos e invertir debido a las dificultades para gestionar los riesgos por los cambios inesperados en el clima.

\section{Figura 7}

Instituciones que ofertan financiamientos al productor.

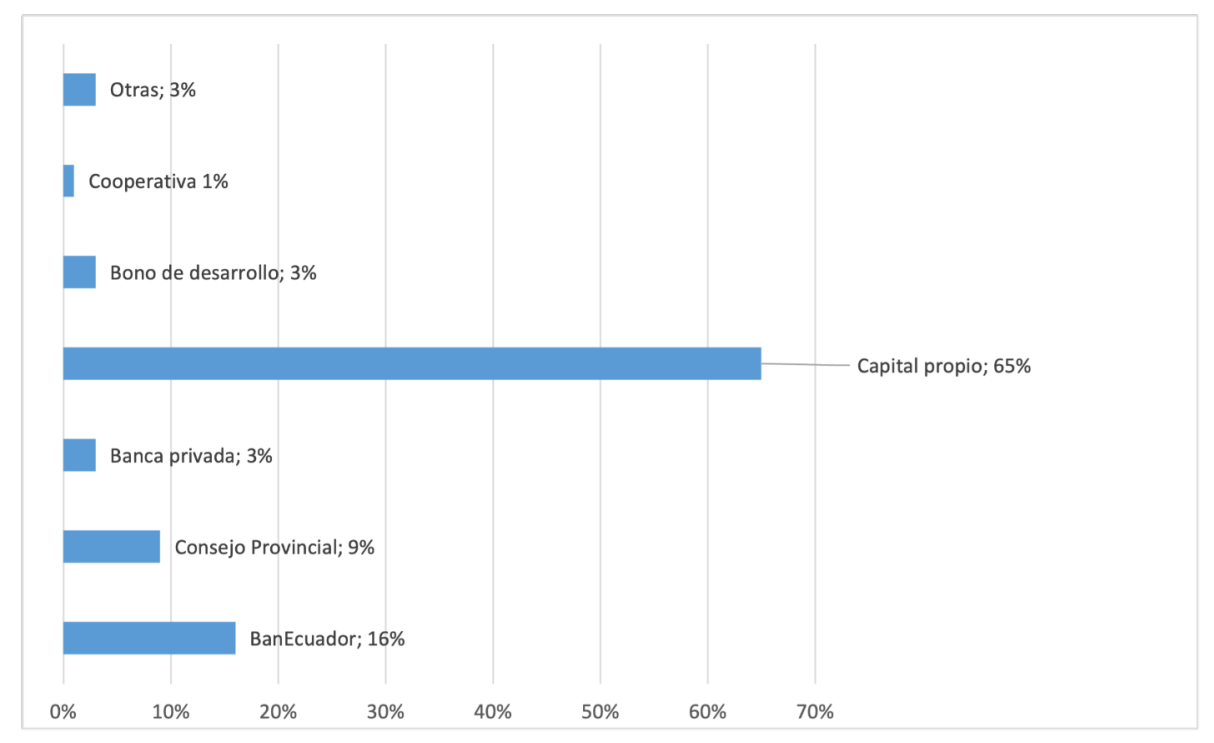

Nota: Elaborado por los Autores.

El $65 \%$ de los encuestados financia su producción con capital propio, el 28\% de los productores busca financiar su producción con apoyo de BanEcuador, Consejo Provincial y el $\mathrm{BDH}$. Un $4 \%$ accede a financiamiento de la banca privada y cooperativas de ahorro y crédito. Las comunidades objeto de estudio, tienen como actividad principal la agricultura y deben ser apoyadas por los diferentes estamentos públicos $\mathrm{y}$ privados, pero ese apoyo es lento y burocrático.

La cultura asociativa del objeto de estudio es débil, prefieren trabajar de manera individual, por miedo a perder la inversión o sus utilidades debido a que no se persigan los mismos objetivos o falta de cooperación entre los agremiados. Diríamos que existe una anti-asociatividad y baja cultura por trabajar en grupos de productores para hacer frente a los intermediarios y los bajos precios que estos pagan por sus productos que al comercializarlos sólo satisfacen sus necesidades de alimentación. La intermediación afecta severamente a los productores. 
Figura 8

Intermediarios en la comercialización.

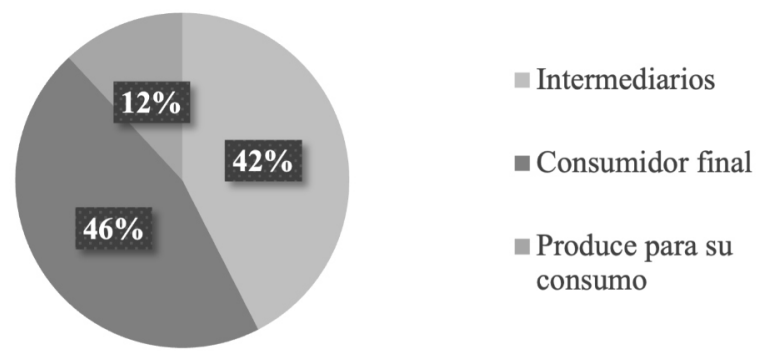

Nota: Elaborado por los Autores.

De acuerdo con estos resultados el $42 \%$ vende su producción a los intermediarios, afectando su cadena de valor, como resultado de la carencia de asociatividad. Los productores esperan en sus parcelas a los intermediarios, quienes pagan precios bajos y, en muchas ocasiones ni siquiera les compran todo el producto, originándoles pérdidas significativas.

En ese sentido, el hecho de que los productores agrícolas obtengan precios bajos en la comercialización que en muchos casos no les permita recuperar la inversión, lleva a repensar el hecho de, si trabajar individualmente tiene incidencia en la decisión de vender a intermediarios, para conocer si existe relación de dependencia entre esas variables se plantea el estadístico Chi-cuadrado de Pearson.

Tabla 4. Prueba de chi-cuadrado ¿Actualmente forma parte de una asociación? ${ }^{*}$ ¿A quiénes les venden su producción?

\begin{tabular}{llll}
\hline & Valor & df & $\begin{array}{l}\text { Significación asintótica } \\
\text { (bilateral) }\end{array}$ \\
\hline Chi-cuadrado de Pearson & $35,346^{\mathrm{a}}$ & 2 & 0,000 \\
Razón de verosimilitud & 43,674 & 2 & 0,000 \\
Asociación lineal por lineal & 27,790 & 1 & 0,000 \\
N de casos válidos & 193 & & \\
\hline
\end{tabular}

a. 1 casillas $(16,7 \%)$ han esperado un recuento menor que 5 . El recuento mínimo esperado es 2,74 .

Este resultado demuestra que, con un nivel de significancia de 0,000 $<0,05 \%$ existe dependencia de las variables, es decir que, el bajo nivel de asociación y el marcado carácter del individualismo en la producción y comercialización hacen que el pequeño productor venda sus unidades de producción a los intermediarios, mostrando poco poder de negociación, haciéndolo un agente tomador de menor precio, lo cual impacta directa y negativamente en su nivel de ingresos. Esto afecta la cadena de valor. La presencia de los intermediarios afecta de una manera más contundente los intereses de los productores, teniendo en cuenta que tanto la utilidad bruta como la rentabilidad son relativamente menores. 


\section{DISCUSIÓN}

La asociatividad es un mecanismo de cooperación entre individuos o empresas pequeñas en donde, según Iguera (2012) cada empresa participante, "manteniendo su independencia jurídica y autonomía gerencial, decide voluntariamente participar en un esfuerzo conjunto con los otros participantes para la búsqueda de un objetivo común" (p.21).

Esta asociatividad surge como mecanismo de cooperación entre organizaciones que están enfrentando problemas internos, así como un proceso de globalización, por ende, es entendida como una forma de acceder a los bienes necesarios para la reproducción amplia de los sujetos y como una forma de hacer sociedad.

No obstante, a lo expresado en relación con las bondades de la asociatividad, para Liendo y Martínez (2001), su viabilidad se ve limitada por algunos factores, siendo el más importante la falta de cultura asociativa en las pequeñas empresas, emprendedores, microempresarios y comerciantes que tradicionalmente han desarrollado un espíritu competitivo y no de cooperación.

A este individualismo comercial, no se escapan los productores de la Parroquia rural El Anegado, lugar de campesinos envejecidos por el trabajo en el campo y en donde los jóvenes estudian o han emigrado. Aquí, el comercio informal y trabajo a jornal han impedido que se fortalezcan alianzas estratégicas para mejorar el sistema productivo. El bajo nivel de producción y productividad debido a que la región depende de la temporada de invierno por las lluvias, el deficiente nivel económico, insuficientes líneas de crédito, escaso nivel organizativo, deficiente sistema de riego, insuficientes canales de comercialización y exiguos controles de precios, les cobran un alto precio a los agricultores que, por años, no pueden mejorar su situación socioeconómica.

La asociatividad desde la cooperación, solidaridad, trabajo en equipo en busca de nuevas alternativas, es una de las soluciones a los problemas que afectan a las Mipymes y OEPS, aún más, en tiempos de pandemia para lograr su sostenibilidad. A pesar de esto, se ha evidenciado en los productores agrícolas de la parroquia El Anegado el miedo al fracaso, el amor al trabajo individual y sin objetivos, un desconocimiento a los beneficios del trabajo en equipo; estos, han sido los factores que han influido en la no aplicación de esta estrategia para potencializar la agricultura y beneficios para el sector campesino.

Actualmente en el Ecuador, existen muchas Mipymes y OEPS, que tienen un tiempo de sobrevivencia corto y limitado a consecuencia de la falta de cultura asociativa y su ineficiencia en la cadena de valor, Hurtado (2017) menciona que esta situación va ligada a su tamaño y dificultades operativas.

En relación con las implicaciones, un aspecto que bien vale la pena resaltar, es el cultural, en algunas regiones los mismos valores, costumbres y tradiciones son pilares importantes para que las personas que constituyen empresas tiendan a creer que mediante la asociatividad se logran más y mejores resultados, si bien, en estos territorios generalmente las industrias crecen más rápidamente y se observa mayor incremento de la economía, lo cual puede verse a pasos más lentos en aquellos territorios donde el entorno empresarial no se orienta a la asociatividad 
(Viloria, 2013).

La asociatividad ayuda a controlar la cadena de valor según Viloria (2013). El hecho de no asociarse hace que el enfoque de la cadena de valor se altere, en el caso de los productores agrícolas, este se inicia cuando el productor combinando los factores productivos cosecha su producto, las limitaciones son variadas y van desde la falta de recursos para obtener las semillas y tecnología, sistema de riego, el capital es prestado lo que no permite que el agricultor tenga independencia en su proceso. Luego el tema de la comercialización es una limitante, el invierno y la transportación, hacen que la producción sea vendida a intermediarios, afectando con esto al agricultor quien en muchos casos no obtiene la rentabilidad esperada. Por otro lado, el consumidor final paga precios altos, quien gana al final es el intermediario, que compra barato $y$ vende a precios altos.

El modelo asociativo destaca que la incorporación a cualquier asociación es voluntaria, ya que ninguna empresa o persona es forzada a integrar un grupo, sino que lo hace cuando tiene la convicción de que puede generarle oportunidades de crecer y mejorar.

Es importante señalar que, si la asociación no puede llevar a cabo una gestión empresarial eficaz, se verá afectada. No se lograrán los objetivos propuestos, así como administrar efectivamente recursos como maquinaria agrícola o sistemas de riego, industrializar la producción o mejorar la comercialización será una utopía. Entonces, la cohesión, la confianza y el compromiso entre socios será más difícil de mantener y aumentará la desconfianza entre socios.
Otro factor resaltante es el proporcionado por Iguera (2012), quien menciona que la asociatividad mantiene la autonomía general de las empresas o personas ya que no se trata de un (Joint Venture), en el cual las decisiones de cada participante están en función de las necesidades de los demás. En el caso de los grupos asociativos, cada participante mantiene su autonomía, y decide qué hacer con los beneficios que obtiene del proyecto.

A partir de estos conceptos, lo ideal es buscar socios que tengan valores comunes y cuyas habilidades se complementen entre sí, de tal manera que, todos tengan algo para contribuir y, llevarse del grupo. De allí, que en función del objetivo que persigue el grupo asociado, pueden generarse relaciones más o menos duraderas; en algunos casos, la asociación puede concluir en la formación de una empresa con personalidad jurídica y patrimonio propio, pero en este caso, ya deja de ser un modelo asociativo.

A través de la asociatividad, se obtiene mayor poder de negociación, mejora el acceso a tecnologías de productos o procesos y a financiamiento y a la vez se comparte riesgos, costos y beneficios. De allí, que es posible afirmar que en el futuro la forma óptima de organización industrial no será ni las pequeñas empresas ni las grandes corporaciones, sino las estructuras en red, que comparten las ventajas de ambas; esto, debido a que las organizaciones en red pueden aprovechar las ventajas de la gran escala, evitando al mismo tiempo los costos generales y administrativos de las grandes organizaciones centralizadas.

Por consiguiente, según Parrales y Rodríguez (2019), el proceso de asociatividad implica 
cambiar actuaciones históricas entre los miembros para poner en acción una nueva sucesión de actividades que permitan desarrollar competencias y habilidades que las vuelvan competitivas y denoten su ventaja comparativa. Lograrlo no es fácil, la costumbre impera ante las demandas actuales, en donde la mayoría de los emprendedores y productores muestran posturas anti-asociativas, dejando en claro que no quieren asociarse.

Las Mipymes y OEPS deben superar barreras culturales o diferencias particulares para aunar esfuerzos por el bienestar de sí misma y de la cadena que crea y trabajar de manera armónica y decidida de manera asociativa. Deben tomarse en serio los compromisos a largo plazo, y no deben verse obstaculizados por la pérdida de su identidad o de la autonomía personal de la empresa, porque no se trata de una fusión de empresas, sino de cooperación. Sólo así se puede formar una verdadera cadena de creación de valor. La asociatividad es una estrategia que permite replantear las condiciones de la comercialización, mediante la búsqueda de canales propios y apropiados en su cadena de valor.

\section{CONCLUSIONES}

Las Mipymes, tienen un papel clave en la generación de empleo y en el desarrollo de las economías nacionales y regionales de cada nación; no obstante, son las grandes empresas las que, dada su escala poseen la discrecionalidad de fijar precios y condiciones en base a sus estructuras de costos y beneficios y discrecionalmente a su ventaja competitiva.

Esta tendencia indica que, cada día es más difícil que este tipo de empresas logren mantenerse y hasta subsistir en el mercado, ya que no sólo se trata de organizaciones compitiendo individualmente frente a otras, sino de grupos organizacionales compitiendo entre sí, obligando a las Mipymes a enfrentarse con la necesidad de replantear su funcionamiento para adaptarse competitivamente a las nuevas exigencias del mercado.

En este contexto se percibe a la asociatividad como una estrategia resultado de una cooperación o coalición de empresas en función de un objetivo en común, en la que cada participante mantiene independencia jurídica y gerencial, las mismas representan una alternativa válida y necesaria para enfrentar los cambios estructurales establecidos a nivel empresarial de manera más eficiente, dado que permite estimular las potencialidades $y$ capacidades individuales de productores, emprendedores, micro, pequeñas y medianas organizaciones, fortaleciéndolas y permitiéndoles enfrentar la competencia, logrando así, un mejor posicionamiento en los mercados. De ahí la necesidad de aliarse para comprar, conseguir financiamiento, negociar con los bancos las tasas de interés, orientando las acciones en lugar de hacia la competencia, hacia la complementación.

Corresponde entonces señalar que la articulación de la cadena de valor con los sistemas asociativos, constituye la base fundamental para el desarrollo de cualquier modelo organizacional, siempre y cuando sus participantes se caractericen por su compromiso, capacidad de cooperación y trabajo conjunto. Dicho proceso dependerá en gran medida del grado de capital social existente entre los potenciales participantes, haciendo hincapié en la confianza, reciprocidad, compromiso, cooperación, entre otros. Dicha 
integración, genera como impacto, el reconocimiento de los esquemas asociativos, entendidos éstos, como una unión organizacional capaz de promover y facilitar el desarrollo de las ventajas competitivas de los conjuntos integrados, contribuyendo de esa forma, con su sostenibilidad, posibilidad de desarrollo y crecimiento de cada país. 
$\begin{array}{lllllll}\text { R } & \text { E } & \text { V } & \text { I } & S & \text { T } & \text { A }\end{array}$

\section{REFERENCIAS}

Alvarado, W. (2016). Cadena de valor Empresarial. Foro Regional sobre Política Industrial-Santa Cruz de la Sierra, 23. https://www.url.edu.gt/ PortalURL/Archivos/56/Archivos/ cadenas.pdf

Arias, F. (2010). Paradigmas de la Investigación Científica. España: Editorial: Luces.

Benitez, C. (2015). Cadena de Valor y su impacto en la asociatividad. Cuaderno de desarrollo económico No 3 Universidad de La Plata, 32.

Padilla, R. (2014). Fortalecimiento de las cadenas de valor como instrumento de la política industrial: Metodología y experiencia de la CEPAL en Centroamérica. CEPAL. https:// repositorio.cepal.org/bitstream/handle/ $\begin{array}{llllllllllll}1 & 1 & 3 & 6 & 2 & / & 3 & 6 & 7 & 4 & 3 & /\end{array}$ S20131092_es.pdf?sequence $=4$ \&isAllowed $=$ y

Clark, J., Teresa de Jesús, M., y Salazar, G. (2017). Competitividad en las Empresas Asociativas Rurales productoras de cacao del cantón Vinces, Provincia de los Ríos-Ecuador. Revista Espacios, 39 (11). https:// www.revistaespacios.com/a18v39n11/ a18v39n11p15.pdf

Hurtado, O. (2007). Las Costumbres de los Ecuatorianos. Quito: Planeta. https:// isbn.cloud/9789978983416/las-costumbresde-los-ecuatorianos/

Iguera, R. (2012). Asociatividad entre PYMES. Revista de Economía de la Universidad del Zulia en Maracaibo, Caracas., 14. https:// www.elmayorportaldegerencia.com/ Documentos/Emprendedores/ \%5BPD\%5D\%20Documentos\%20\%20Asociatividad\%20en\%20pymes.pdf
Katzenbach, J. R. and Smith, D.K. (1993), The Wisdom of Teams: Creating the High-performance Organization, Harvard Business School, Boston.

Liendo, M. y Martínez, A. (2001). Asociatividad una alternativa para el desarrollo y crecimiento de las Pymes. Sextas Jornadas "Investigaciones en la Facultad" de Ciencias Económicas y Estadística. Instituto de Investigaciones Económicas, Escuela de Economía. Universidad Nacional de Rosario. https:// hdl.handle.net/2133/8044

Otoya, J. J. (2005). Características e importancia de la pyme en nuestra economía. La PYME en el Perú, 33. http://cendoc.esan.edu.pe/ fulltext/e-journals/PAD/7/arbulu.pdf

Parrales, M. y Rodríguez, K. (2019). Asociatividad y cadena de valor frente al crecimiento económico. II Congreso Internacional de Gerencia y Gestión Productiva \#CI2GP 2019. Pontificia Universidad Católica del Ecuador, sede Manabí. congresospucem@pucem.edu.ec

Porter, M. (1995). Las PYMES. Asociatividad y su impacto en el mundo competitivo de las organizaciones. Cuaderno de desarrollo económico No 3 Universidad de La Plata, 11. https://www.redalyc.org/pdf/ 818/81803203.pdf

Ron Amores, R., y Sacoto Castillo, V. (30 de Junio de 2017). Las PYMES ecuatorianas: su impacto en el empleo como contribución del PIB, PYMES al PIB total. ESPACIOS, 38(53), 15. https://www.revistaespacios.com/ 


\section{a17v38n53/a17v38n53p15.pdf}

Rosales, D. (2016). La competitividad de las PYMES. Revista de Economía de Perú. Seminario Internacional Desafíos, 24. https:// www.cepal.org/es/discursos/seminariointernacional-desafios-la-planificacionmarco-la-agenda-2030-ods

Swans, G. (2015). Asociatividad y Productividad un eje para el desarrollo de la competitividad. Artículo empresarial del Boletín Centro de Información y documentación de-El Salvador, 32.

Viloria, M. (2013). Asociatividad Empresarial. PRODES Programa de la Universidad de España, 18. 